\title{
Diversité spatio-temporelle des glossines le long du fleuve Comoé en Côte d'Ivoire
}

\author{
Vincent Djohan ${ }^{1,2 *}$ Dramane Kaba ${ }^{1}$ Jean-Baptiste Rayaissé ${ }^{3}$ \\ Ernest Salou ${ }^{3}$ Bamoro Coulibaly ${ }^{1}$ Fabien Dofini ${ }^{3}$ \\ Koffi A.M. Kouadio ${ }^{1}$ Philippe Solano ${ }^{4}$ Hervé Menan ${ }^{2}$
}

\begin{abstract}
Mots-clés
Glossina, biodiversité,

trypanosomose, fleuve Comoé, Côte d'Ivoire
\end{abstract}

Submitted: December 20, 2014; Accepted: July 17, 2015; Published: November 20, 2015
1. Institut Pierre Richet / INSP, BP 1500 Bouaké 01, Côte d'Ivoire.

2. Université Félix Houphouët Boigny, Abidjan, Côte d'Ivoire.

3. Cirdes, Bobo-Dioulasso, Burkina Faso.

4. IRD UMR Intertryp, IRD / Cirad, Monpellier, France.

* Auteur pour la correspondance

Tél. : +22540740401 - vincentdjohan1@yahoo.fr

\section{Résumé}

Afin d'étudier la diversité spatio-temporelle des glossines vectrices de trypanosomoses en Côte d'Ivoire, des enquêtes ont été réalisées sur le fleuve Comoé dans trois sites localisés dans des zones écoclimatiques différentes : au nord, au centre et au sud. Elles ont eu lieu en saisons sèche et pluvieuse. Pour chaque site et saison, les glossines ont été capturées pendant cinq jours consécutifs à l'aide de pièges biconiques disposés suivant des transects perpendiculaires au fleuve Comoé. A Kafolo, en savane, trois espèces et sous-espèces ont été capturées indépendamment de la saison: Glossina palpalis gambiensis, G. tachinoides et G. medicorum. A Aboisso-Comoé et à Groumania, respectivement en zone de forêt et de transition forêt-savane, seule G. palpalis palpalis a été capturée. La densité apparente par piège, toutes espèces confondues, a été plus importante à Kafolo qu'à Groumania et à Aboisso-Comoé. Elle a été de 9,48 glossines $(\mathrm{gl}) /$ piège/jour (écart-type $[\mathrm{ET}]=26,30$ ) à Kafolo, de $0,79 \mathrm{gl} /$ piège/ jour $(\mathrm{ET}=2,65)$ à Groumania et de $0,18 \mathrm{gl} /$ piège/jour $(\mathrm{ET}=0,58)$ à AboissoComoé en saison sèche. En saison pluvieuse, elle a été de 3,64 gl/piège/jour $(\mathrm{ET}=9,76)$ à Kafolo et de 1,42 gl/piège/jour $(\mathrm{ET}=2,35)$ à Groumania. Ces résultats montrent que la diversité des espèces de glossines le long du fleuve Comoé est fonction de la conservation du biotope, ainsi que l'illustre la disparition progressive de certaines espèces comme G. morsitans submorsitans qui n'a pas été capturée au cours de cette étude. Nous confirmons également que seul le vecteur majeur de la trypanosomiase humaine africaine, G. palpalis s.l., est capable de s'adapter à I'homme.

- Pour citer cet article : Djohan V., Kaba D., Rayaissé J-B, Salou E., Coulibaly B, Dofini F., Kouadio K.A.M., Solano P., Menan H., 2015. Spatial and temporal diversity of tsetse flies along Comoe River in Côte d'Ivoire [in French]. Rev. Elev. Med. Vet. Pays Trop., 68 (1): 39-44

\section{INTRODUCTION}

Les glossines, vecteurs cycliques des trypanosomoses en Afrique subsaharienne, sont responsables d'une morbidité et d'une mortalité importantes chez l'homme et le bétail (Leak, 1999 ; Courtin et al., 2009). Les trypanosomoses africaines, humaines (THA) et animales (TAA), constituent de ce fait une contrainte d'ordre social et économique pour le développement de l'Afrique au sud du Sahara (Molyneux et al., 2010). C'est dans le but d'éradiquer ces pathologies et leurs vecteurs du continent africain qu'est née, sous l'égide de l'Union africaine, la Pan African Tsetse and Trypanosomosis Eradication Campaign (Pattec), une initiative panafricaine. Celleci vise la mise en œuvre d'une approche régionale pour l'élimination des tsé-tsé par l'usage de méthodes de lutte appropriées afin de créer, à terme, des zones indemnes de glossines (Kabayo, 2002). 
De son côté, l'organisation mondiale de la santé (OMS) a initié un programme d'élimination de la THA (Simarro et al., 2008). Pour la réalisation des objectifs de la Pattec, la lutte antivectorielle semble être l'une des meilleures stratégies à employer au vu des différents défis liés à la résistance aux trypanocides et au contrôle du réservoir animal (Simarro et al., 2008). La réalisation efficace de cette lutte nécessite de mieux comprendre le comportement des vecteurs dans leurs différents biotopes ainsi que les relations existant entre les vecteurs eux-mêmes (Courtin et al., 2009 ; Courtin et al., 2010) C'est ce qui justifie la présente étude qui visait à déterminer la diversité des glossines riveraines le long du fleuve Comoé, en Côte d'Ivoire, en fonction des saisons et des zones écoclimatiques, ainsi que leur comportement de dispersion dans cet environnement.

\section{MATERIEL ET METHODES}

\section{Zones d'étude}

Ce travail a été effectué en Côte d'Ivoire, le long du fleuve Comoé, dans trois zones écoclimatiques différentes : la zone de forêt au sud, la zone de transition forêt-savane au centre et la zone de savane au nord. Cela a permis de travailler dans des biotopes variés, favorisant une meilleure évaluation de la biodiversité des glossines. Des transects ont été réalisés sur ces trois sites d'étude, le long du fleuve (figure 1).

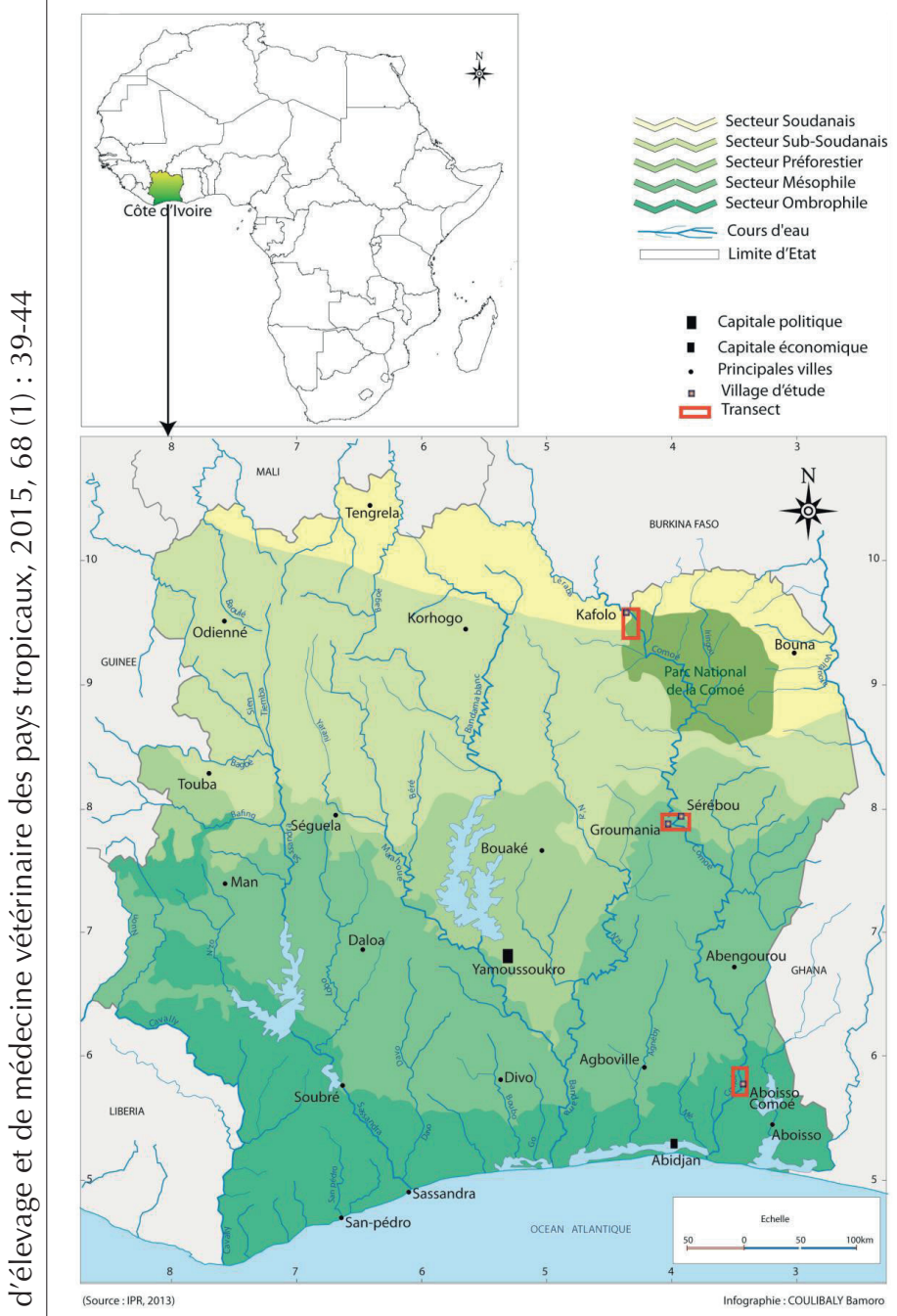

Figure 1 : localisation des sites d'étude le long du fleuve Comoé en Côte d'Ivoire.
Un transect a été réalisé au sud, dans la forêt classée de la Yaya, à Aboisso-Comoé dans le département d'Alépé ( $5^{\circ} 46^{\prime}$ de lat. $\mathrm{N}$ et $3^{\circ} 10^{\prime}$ de long. O). Les pluies y sont abondantes avec des précipitations avoisinant $1750 \mathrm{~mm}$ et une température moyenne de $26,6{ }^{\circ} \mathrm{C}$ (Anonyme, 2005). La végétation est très luxuriante et typique du domaine guinéen, avec une forêt dense sempervirente à Eremospatha macrocarpa et Diospyros mannii (Guillaumet et Adjanohoun, 1971), dégradée par endroit par des cacaoyères, des caféières, des complexes culturaux et des exploitations vivrières.

Au centre, dans la zone de transition forêt-savane, le second transect a été réalisé entre les villages de Groumania et de Sérébou qui se situent dans la zone de transition entre Glossina palpalis gambiensis et G. p. palpalis (Challier et al., 1983 ; Nekpeni et al., 1989), Sérébou constituant la limite septentrionale de capture de G. p. palpalis (Challier et al., 1983). Le site est situé à $8^{\circ} 23^{\prime}$ de lat. $\mathrm{N}$ et $4^{\circ} 26^{\prime}$ de long. O. Le climat est caractérisé par l'alternance annuelle d'une saison des pluies et d'une saison sèche. Les précipitations avoisinent $1300 \mathrm{~mm}$ et la température moyenne annuelle est de $26,8^{\circ} \mathrm{C}$ (Anonyme, 2005). Aubrevillea kerstingii et Khaya grandifolia forment la lisière des forêts semi-décidues et l'essentiel des bosquets en savane (Guillaumet et Adjanohoun, 1971). La végétation se compose d'une mosaïque forêt-savane bordant la forêt galerie, avec par endroits une végétation de savane arborée humide côtoyant des accrus d'Azadirachta indica (neem). Des plantations villageoises d'Anacardium occidentale ainsi que de petites exploitations de cultures vivrières sont présentes sur le site.

Le troisième transect a été réalisé dans le parc national de la Comoé à l'extrême nord du pays, à la frontière avec le Burkina Faso, près du village de Kafolo ( $9^{\circ} 35^{\prime}$ de lat. N et $5^{\circ} 12^{\prime}$ de long. O), dans la sous-préfecture de Kong (Anonyme, 2005). Le climat y est caractérisé par une courte saison des pluies et une longue saison sèche. Les précipitations moyennes annuelles atteignent $1200 \mathrm{~mm}$ et la température moyenne annuelle est de $26,7^{\circ} \mathrm{C}$ (Anonyme, 2005). La végétation comprend une forêt galerie bordant le fleuve Comoé et des savanes arbustives, composées d'arbustes et de hautes herbes. La forêt galerie est relativement étroite, ne dépassant que rarement les 200 mètres de large. L'ensemble du parc national de la Comoé appartient au domaine soudanais et les formations végétales qui le caractérisent sont les savanes, les forêts claires et les forêts denses sèches. La physionomie des savanes est déterminée par la densité des espèces ligneuses associées au tapis herbacé et par leur taille. Les forêts claires délimitent des surfaces dont le couvert arboré a une taille moyenne de 15 mètres de hauteur. Les espèces caractéristiques sont Isoberlinia doka, Daniellia oliveri, Pterocarpus erinaceus, Uapaca togoensis, Parkia biglobosa et Vitellaria paradoxa. Les forêts denses sèches sont représentées surtout par les forêts galeries qui bordent les cours d'eau. La hauteur des arbres peut y atteindre 30 mètres. Les espèces caractéristiques sont Antiaris africana, Milicia excelsa, Anogeissus leiocarpus, Cola cordifolia et Celtis zenkeri. Le parc national de la Comoé est une zone protégée. La faune comprend entre autres des ongulés (buffles, cobes, céphalophes, phacochères), des varans, des singes et des félins (Guillaumet et Adjanohoun, 1971).

\section{Echantillonnage des glossines et étude de leur distribution en fonction des biotopes}

Cette étude a été réalisée au moyen de sondages entomologiques effectués à l'aide de pièges biconiques (Challier et al., 1977) disposés sous forme de transects. Sur chaque site, cinq transects distants l'un de l'autre d'un kilomètre au minimum ont été disposés perpendiculairement au cours d'eau. Chaque transect était composé de cinq pièges, posés à intervalle de 200 mètres, partant de la berge et s'étendant en profondeur jusque dans la forêt ou la savane. Au total, 25 pièges ont été posés par site (figure 2). 


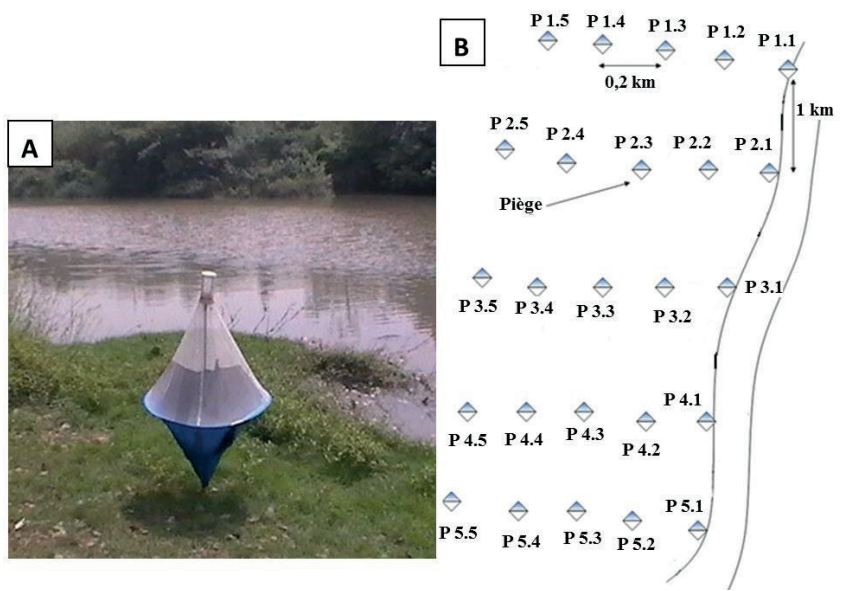

Figure 2 : piège biconique $(A)$ et disposition des transects le long du fleuve Comoé (B) en Côte d'Ivoire.

Les sondages ont été réalisés en saison sèche (du 26 janvier au 10 mars 2012) et en saison pluvieuse (du 22 octobre au 14 novembre 2012), sauf à Aboisso-Comoé où ils n'ont été réalisés qu'en saison sèche. Chaque série de sondage a duré cinq jours consécutifs avec une collecte quotidienne des cages de capture. Les glossines capturées ont été comptabilisées par localité, par piège, par espèce et par sexe en fonction des saisons. Tous les points de piégeage ont été géoréférencés à l'aide d'un géopositionnement par satellite (GPS) afin d'établir une cartographie de la distribution des glossines dans les zones d'étude. La comparaison des résultats en fonction des saisons a permis d'identifier les préférences des différentes espèces de glossines pour le biotope et d'y apporter des éléments d'explication.

\section{Analyses statistiques}

La diversité spécifique dans les différents biotopes a été approchée par la détermination de plusieurs indices de biodiversité, l'indice d'équitabilité de Pielou (Pielou, 1966) et l'indice de diversité de Hill (Hill, 1973). L'indice d'équitabilité, également appelé indice d'équirépartition, varie de 0 à 1 . Il est maximal quand les espèces ont des abondances identiques dans le peuplement et minimal quand une seule espèce domine le peuplement. Il est insensible à la richesse spécifique et très utile pour comparer les dominances potentielles entre stations ou entre dates d'échantillonnage. L'indice de diversité de Hill mesure l'abondance proportionnelle en associant l'indice de Shannon-Weaver (plus sensible aux effectifs des espèces rares) et l'indice de Simpson (plus sensible aux effectifs des espèces abondantes) : l'indice de Hill semble ainsi être le plus synthétique. Plus l'indice s'approche de la valeur 1, plus la diversité est faible. Toutefois, afin de faciliter l'interprétation, on utilise souvent l'indice 1-Hill, où la diversité maximale est représentée par la valeur 1, et la minimale par la valeur 0 .

Ces indices visent à permettre une meilleure appréciation de la diversité spécifique des glossines dans les différents transects étudiés. Les données ont été saisies sur Excel et analysées avec le logiciel R (version 2.8) (Ihaka et Gentleman, 1996). La comparaison des densités apparentes par piège (DAP) (facteur aléatoire) avec la position du piège dans le milieu (facteur fixe) en fonction des saisons (facteur fixe) a été réalisée en utilisant les modèles linéaires mixtes avec effets aléatoires à partir du logiciel SPSS version 16 avec une valeur de p inférieure à 0,05 .

\section{RESULTATS}

$\mathrm{Au}$ total, quatre espèces et sous-espèces de glossines, à savoir Glossina palpalis palpalis, G. p. gambiensis, G. tachinoides et G. medicorum, ont été capturées au cours des enquêtes entomologiques ; elles étaient diversement réparties dans les différents biotopes. G. p. palpalis a été capturée au sud (Aboisso-Comoé), en saison sèche, et au centre (Groumania) pendant les saisons sèche et pluvieuse. G. p. gambiensis, G. tachinoides et $G$. medicorum ont, quant à elles, été capturées au nord (Kafolo) pendant les deux saisons. La densité apparente, toutes espèces confondues, était, en saison sèche, de 9,48 glossines ( $\mathrm{gl}$ )/piège/jour (écart-type $[\mathrm{ET}]=26,30)$ à Kafolo, de 0,79 gl/piège/jour $(\mathrm{ET}=2,65)$ à Groumania et de $0,18 \mathrm{gl} /$ piège/jour $(\mathrm{ET}=0,58)$ à Aboisso-Comoé. En saison pluvieuse, elle était de $3,64 \mathrm{gl} /$ piège/jour $(\mathrm{ET}=9,76)$ à Kafolo et de 1,42 gl/piège/jour $(\mathrm{ET}=2,35)$ à Groumania.

Les indices de diversité n'ont pu être calculés qu'à Kafolo puisque seule G. p. palpalis a été capturée à Groumania et à Aboisso-Comoé. Ces indices ont été relativement identiques au cours des deux saisons (tableau I). Toutefois, l'analyse a montré qu'en saison sèche, une espèce ( $G$. tachinoides) dominait le peuplement en savane. Par contre, dans la galerie longeant la rivière, il n'y avait pas de dominance spécifique ce qui s'est traduit par la présence de plusieurs espèces de glossines, toutes s'adaptant bien à la galerie forestière. En saison pluvieuse en revanche, que ce soit en savane ou dans la galerie, les tsé-tsé ont été relativement bien réparties. L'indice de diversité de Hill (1-Hill) a montré globalement une

\section{Tableau I}

Indices de diversité selon les sites le long du fleuve Comoé et les saisons en Côte d'ivoire

\begin{tabular}{|c|c|c|c|c|c|}
\hline \multirow[t]{3}{*}{ Indices de diversité } & \multicolumn{5}{|c|}{ Sites } \\
\hline & \multicolumn{2}{|c|}{ Kafolo } & \multicolumn{2}{|c|}{ Groumania } & \multirow{2}{*}{$\begin{array}{c}\text { Aboisso-Comoé } \\
\text { Saison sèche }\end{array}$} \\
\hline & Saison sèche & Saison pluvieuse & Saison sèche & Saison pluvieuse & \\
\hline Abondance (nb. de mouches capturées) & 1185 & 455 & 99 & 179 & 23 \\
\hline Nb. d'espèces présentes & 3 & 3 & 1 & 1 & 1 \\
\hline Equitabilité & 0,494 & 0,460 & NA & NA & NA \\
\hline $1-\mathrm{Hill} *$ & 0,117 & 0,146 & NA & NA & NA \\
\hline
\end{tabular}

* 1 = diversité maximale, 0 = diversité minimale

NA : non applicable 
diversité spécifique faible avec des tendances inverses en saison sèche et en saison pluvieuse. En saison sèche, la diversité était plus importante dans la galerie que dans la savane, l'inverse étant observé en saison des pluies avec des rapports entre les indices compris entre 2 et 3 (tableau II).

De la zone de forêt à la zone de savane, la répartition des glossines dans les sites de capture était de moins en moins homogène. En forêt, à Aboisso-Comoé, les glossines étaient bien réparties le long des transects alors qu'en savane (Kafolo), elles ont essentiellement été capturées dans la galerie forestière, et ce, au cours des deux saisons (figure 3). Seule G. medicorum, inféodée à un bosquet, a

\section{Tableau II}

Indices de diversité selon les biotopes le long du fleuve Comoé et les saisons à Kafolo en Côte d'ivoire

\begin{tabular}{lcccc}
\hline Indices de diversité & \multicolumn{4}{c}{ Kafolo } \\
\cline { 2 - 3 } & \multicolumn{3}{c}{ Saison sèche } & Saison pluvieuse \\
\cline { 2 - 3 } & Rive & Savane & Rive & Savane \\
& 1118 & 67 & 386 & 69 \\
$\begin{array}{l}\text { Abondance } \\
\text { (nb. de mouches capturées) }\end{array}$ & 3 & 2 & 3 & 3 \\
$\begin{array}{l}\text { Nb. d'espèces présentes } \\
\text { Equitabilité }\end{array}$ & 0,509 & 0,111 & 0,595 & 0,569 \\
1-Hill * & 0,113 & 0,046 & 0,051 & 0,183
\end{tabular}

* 1 = diversité maximale, 0 = diversité minimale
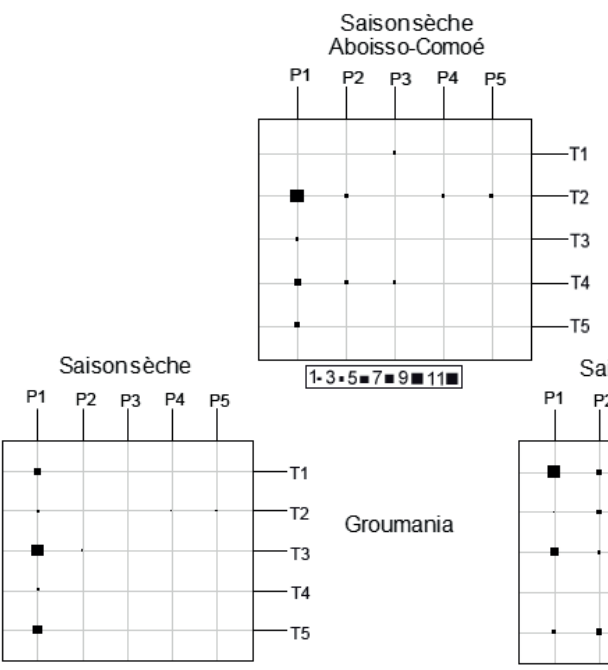

5.15.25!35-45!
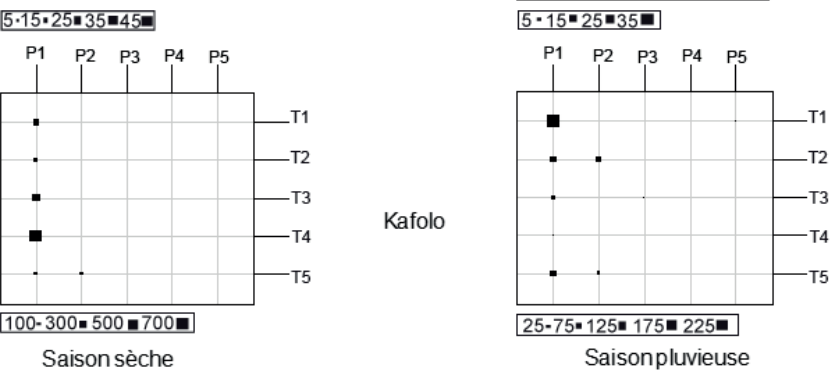

Figure 3 : abondance globale des glossines par transect et par piège en fonction des saisons et des sites d'étude le long du fleuve Comoé en Côte d'Ivoire. été capturée en saison des pluies hors de la galerie forestière sur ce site. Dans la zone de transition forêt-savane (Groumania), une situation intermédiaire a été observée avec une répartition plus homogène en saison pluvieuse (figure 3) sauf au niveau des trois derniers pièges du transect $\mathrm{T} 5$, placés dans les accrus d'Azadirachta indica. En saison sèche, les glossines n'ont guère été observées à partir de 200 mètres au-delà de la galerie, sauf dans le transect T2 où elles ont été capturées à 600 et 800 mètres de la galerie. Cette partie du transect T2 comprenait quelques bosquets et des étangs non asséchés.

A Aboisso-Comoé et à Groumania, en saison sèche, G. p. palpalis a été capturée de façon significativement plus importante dans la galerie forestière que dans les autres paysages. En saison pluvieuse à Groumania, bien que les captures aient été plus importantes dans la galerie, la différence a été moins grande, et même statistiquement non significative, avec les captures réalisées en savane (figure 4).
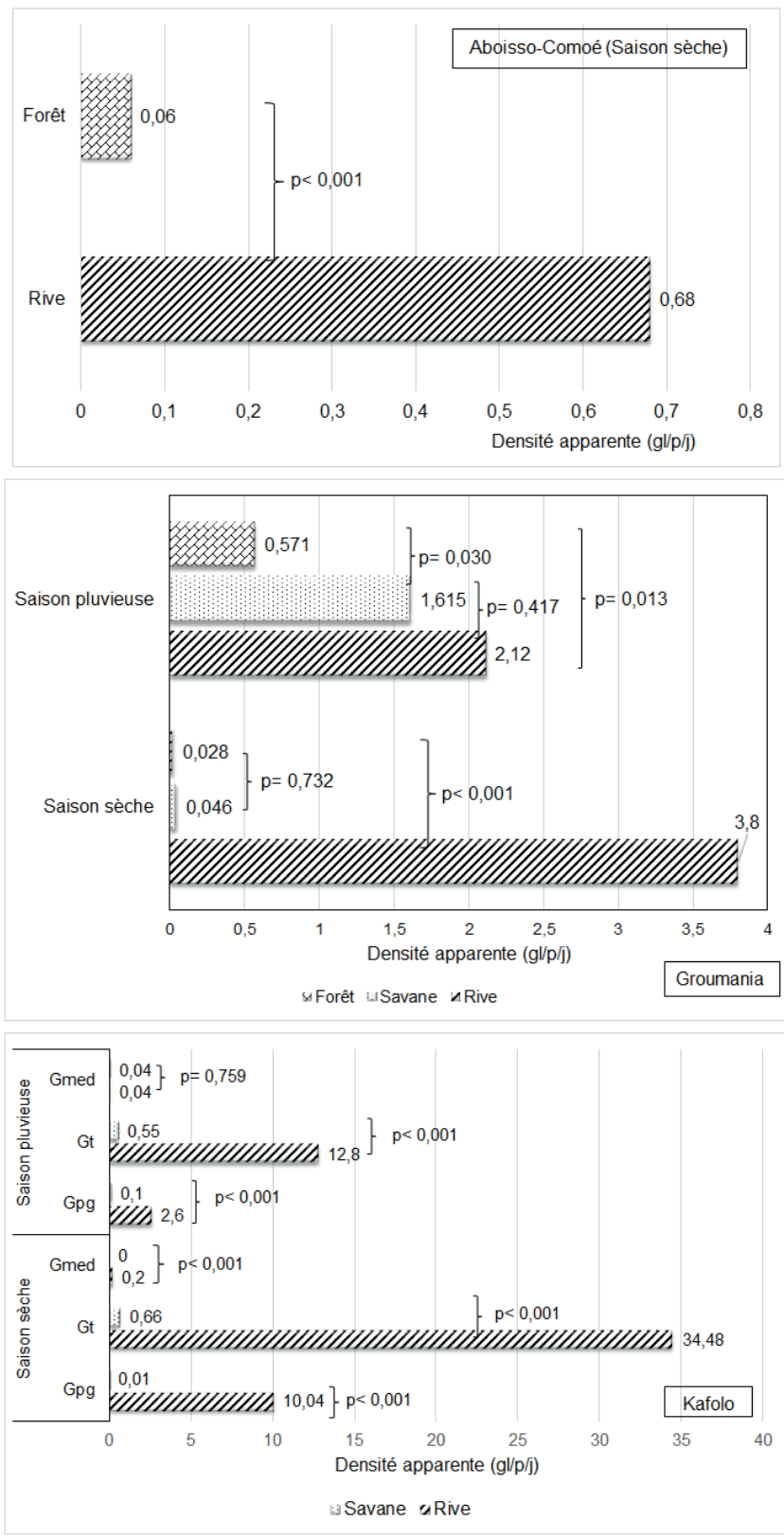

Figure 4 : comparaison des densités apparentes par piège des espèces de glossines en fonction des lieux de capture le long du fleuve Comoé en Côte d'Ivoire. 


\section{DISCUSSION}

Le transect nord-sud réalisé le long du fleuve Comoé traverse différents faciès bioclimatiques de la Côte d'Ivoire (savane, transition forêt-savane et forêt). Parmi les dix espèces et sous-espèces de glossines recensées il y a trente ans dans le pays (Laveissière et Challier, 1981), quatre ont été capturées lors de cette étude, dans des biotopes qui leur sont habituels (Challier, 1968 ; Laveissière et Challier, 1981 ; Challier et al., 1983). Une certaine diversité spécifique a été observée à Kafolo dans le parc national de la Comoé. Cependant, de manière surprenante, G. m. submorsitans, qui est une espèce de savane, n'a pas été capturée lors de cette étude pourtant menée dans un parc national. Cette absence pourrait s'expliquer par la disparition de la faune sauvage à laquelle elle est inféodée. Cette espèce, ainsi que les autres glossines du groupe morsitans, tendraient à disparaître spontanément quand les mammifères font défaut (Rodhain, 1926 ; Gouteux et al., 1994 ; Rayaissé et al., 2009). Or, après la crise postélectorale de 2010, le parc national de la Comoé est resté sans surveillance, à la merci des braconniers qui y ont décimé la faune. Le recul de G. m. submorsitans est ainsi probablement lié à l'anthropisation de son biotope et à ses conséquenses écologiques que sont notamment le déboisement et le braconnage (Rodhain, 1926 ; Omoogun et al., 1991). Enfin, la faible efficacité du piège biconique vis-à-vis de G. m. submorsitans (Rayaissé et al., 2012) pourrait également expliquer l'absence de capture en cas de faible densité résiduelle de cette espèce dans le parc de la Comoé.

La diversité des espèces de glossines a été faible dans notre étude. Le transect réalisé à Aboisso-Comoé dans la forêt classée de la Yaya n'a permis de capturer que G. p. palpalis, espèce à la fois riveraine et anthropophile. Cette zone forestière est le refuge habituel de G. pallicera, G. nigrofusca et G. fusca (Gouteux et al., 1981 ; Laveissière et Challier, 1981). La présence de G. p. palpalis seule pourrait être due à l'existence de planteurs clandestins qui y ont réalisé des vergers et des plantations de café et de cacao. Cette espèce, contrairement aux espèces des groupes morsitans et fusca qui ont tendance à disparaître dès que les densités humaines augmentent (Reid et al., 2000), est en effet plus résiliente, capable de s'adapter aux environnements péridomestiques (Baldry, 1964) et même au milieu urbain (Allou et al., 2009).

A Kafolo, G. tachinoides a dominé les captures réalisées en savane (c'est-à-dire hors de la galerie forestière). Elle semblait être l'espèce la mieux adaptée à cet environnement alors que les autres espèces (G. p. gambiensis et G. medicorum) demeuraient dans la galerie forestière. En saison pluvieuse comme en saison sèche, la répartition relativement homogène des différentes espèces de glossines dans la galerie a été la conséquence d'un climat plus clément, d'un couvert végétal permanent et de la présence d'hôtes nourriciers. La galerie demeure le lieu le plus sûr pour les glossines. Hors de la galerie, la température élevée et l'absence de lieux de repos favorables, constituaient des freins à la dispersion des glossines.

Au Sud, à Aboisso-Comoé, les pluies sont fréquentes et l'humidité relative élevée. La température moyenne et l'humidité relative varient peu quelle que soit la saison (Anonyme, 2005). Les glossines pouvaient donc se disperser aisément, profitant de conditions climatiques favorables, et n'étaient pas inféodées à la galerie. En revanche, à Groumania, la culture de noix de cajou et les cultures vivrières menaçaient gravement la galerie forestière qui ne dépassait guère 10 mètres de large à certains endroits. Le couvert étant réduit, les mouches se réfugiaient pratiquement toutes dans la galerie pendant la saison sèche. En saison pluvieuse, aucune tsé-tsé n'a été capturée dans la plantation d'Azadirachta indica qui pourtant offrait un bon couvert. Il s'agit d'une plante dont les effets insectifuges ont été montrés au laboratoire sur G. fuscipes fuscipes (Makoundou et al.,
1995). L'absence de glossines dans les accrus d'A. indica serait due aux effets répulsifs de cette plante sur les tsé-tsé.

\section{CONCLUSION}

La lutte contre les vecteurs de trypanosomoses humaine et animale nécessite d'actualiser les données sur leur répartition géographique et leur écologie afin d'adapter outils et méthodes. Des glossines pourraient avoir disparu de certains environnements alors que d'autres, qui n'y étaient pas, les coloniseraient. La répartition des glossines est donc dynamique et dépend de facteurs biotiques et abiotiques, dont certains sont influencés par l'homme. La répartition des glossines ne semble pas avoir évolué depuis les travaux de Laveissière et Challier il y a plus de trente ans au centre et au nord du pays (Laveissière et Challier, 1981). Toutefois, dans la zone forestière du sud, la pression démographique, entraînant une anthropisation accrue des forêts, favorise la disparition de certaines espèces de glossines qui y sont inféodées. Seules persisteront les espèces résilientes à l'anthropisation comme G. p. palpalis qui est, de plus, le vecteur majeur de la trypanosomose humaine africaine en Côte d'Ivoire.

\section{Remerciements}

Nous tenons à remercier les chercheurs et techniciens de l'IPR, du Cirdes et de l'UMR Intertryp pour leur appui technique, ainsi que les autorités administratives et coutumières des localités visitées, sans oublier les guides et les aides. Cette étude a été en grande partie réalisée grâce au financement de l'IRD à travers la JEAI Ecovectryp.

\section{REFERENCES}

Allou K., Acapovi-Yao G., Kaba D., Bosson-Vanga H., Solano P., N'goran K.E., 2009. Chorologie et infection par les trypanosomes de Glossina palpalis palpalis dans la forêt du Banco et ses reliques, Abidjan (Côte d'Ivoire). Parasite, 16 (4) : 289-295

Anonyme, 2005. Départements et districts de Côte d'Ivoire. Office ivoirien du tourisme et de l'hôtellerie, Le groupe intercommunication, Abidjan, Côte d'Ivoire, 420 p.

Baldry D.A., 1964. Observations on a close association between Glossina tachinoides and domestic pigs near Nsukka, Eastern Nigeria. II. Ecology and trypanosome infection rates in G. tachinoides. Ann. Trop. Med. Parasitol., 58 (1): 32-44

Challier A., 1968. Ecologie et biologie de Glossina palpalis gambiensis Vanderplank dans une zone de savanes d'Afrique occidentale. Cah Orstom, Sér. Entomol. Méd. Parasitol., 6 (3-4) : 247-255

Challier A., Eyraud M., Lafaye A., Laveissière C., 1977. Amélioration du rendement du piège biconique pour glossines (Diptera, Glossinidae) par l'emploi d'un cône inférieur bleu. Cah. Orstom, Sér. Entomol. Méd. Parasitol., 15 (3) : 283-286

Challier A., Gouteux J.-P., Coosemans M., 1983. La limite géographique entre les sous-espèces Glossina palpalis palpalis (Rob-Desv.) et Glossina palpalis gambiensis Vanderplanck (Diptera, Glossinidae) en Afrique occidentale. Cah. Orstom, Sér. Entomol. Méd. Parasitol., 21 (4) : 207-220

Courtin F., Rayaissé J.-B., Tamboura I., Serdébéogo O., Koudougou Z., Solano P., Sidibé I., 2010. Updating the northern tsetse limit in Burkina Faso (1949-2009): Impact of global change. Int. J. Environ. Res. Public Health, 7 (4): 1708-1719

Courtin F., Sidibé I., Rouamba J., Jamonneau V., Gouro A., Solano P., 2009. Impacts des évolutions démographiques et climatiques sur la répartition spatiale des hommes, des tsé-tsé et des trypanosomoses en Afrique de l'Ouest. Parasite, 16 (1) : 3-10

Gouteux J.-P., Blanc F., Pounekrozou E., Cuisance D., Mainguet M., D’Amico F., Le Gall F., 1994. Tsé-tsé et élevage en République centrafricaine : le recul de Glossina morsitans submorsitans (Diptera, Glossinidae). Bull. Soc. Pathol. Exot., 87 : 52-56

Gouteux J.-P., Mondet B., Poinar G.O. Jr, 1981. Ecologie des glossines en secteur pré-forestier de Côte-d'Ivoire. 1. Parasitisme par Hexamermis glossinae (Nematoda, Mermithidae). Cah. Orstom, Sér. Entomol. Méd. Parasitol., 19 (4) : 285-295 
Guillaumet J.-L., Adjanohoun E., 1971. La végétation de la Côte d'Ivoire. In : Le milieu naturel de la Côte d'Ivoire (Eds. Avenard J.-M., Eldin M., Girard G., Sircoulon J., Touchebeuf de Lussigny P., Guillaumet J.-L., Adjanohoun E., Perraud A.). Orstom, Paris, France, 161-263

Hill M.O., 1973. Diversity and evenness: A unifying notation and its consequences. Ecology, 54 (2): 427-432

Ihaka R., Gentleman R., 1996. R: A language for data analysis and graphics. J. Comput. Graph. Stat., 5 (3): 299-314

Kabayo J.P., 2002. Aiming to eliminate tsetse from Africa. Trends Parasitol., 18 (11): 473-475

Laveissière C., Challier A., 1981. La répartition des glossines en Côte d'Ivoire. Cartes à 1/2 $000000^{\mathrm{e}}$ et notice explicative. Orstom, Paris, France, $33 \mathrm{p}$.

Leak S.G.A., 1999. Tsetse biology and ecology: Their role in the epidemiology and control of trypanosomosis. CABI, Wallingford, UK, $551 \mathrm{p}$.

Makoundou P.B., Cuisance D., Duvallet G., Guillet P., 1995. Laboratory testing of the effects of a natural insecticide made with neem extracts (Azadirachta indica A. Juss) on Glossina fuscipes fuscipes Newstead, 1910 (Diptera: Glossinidae) [in French]. Rev. Elev. Med. Vet. Pays Trop., 48 (4): 339-345

Molyneux D., Ndung'u J., Maudlin I., 2010. Controlling sleeping sickness - "When will they ever learn?". PLoS Negl. Trop. Dis., 4 (5): e609, doi:10.1371/journal.pntd.0000609

\section{Summary}

Djohan V., Kaba D., Rayaissé J.-B., Salou E., Coulibaly B., Dofini F., Kouadio K.A.M., Solano P., Menan H. Spatial and temporal diversity of tsetse flies along Comoe River in Côte d'Ivoire

To investigate the spatial and temporal diversity of tsetse flies, vectors of trypanosomosis, in Ivory Coast, surveys were conducted on the Comoe River in three sites located in different ecoclimatic zones: north, center and south. They were carried out during the dry and rainy seasons. For each site and season, the flies were captured during five consecutive days in biconical traps arranged in transects perpendicular to the Comoe River. In Kafolo in savanna, three species and subspecies were captured regardless of the season: Glossina palpalis gambiensis, G. tachinoides and G. medicorum. In Aboisso-Comoe and Groumania, in forest and forest-savanna transition areas, respectively, only G. palpalis palpalis was captured. The apparent density per trap, all species combined, was higher in Kafolo than in Groumania and Aboisso-Comoe. It was 9.48 tsetse flies (tf)/trap/day (standard deviation $[S D]=26.30$ ) in Kafolo, $0.79 \mathrm{tf} /$ trap/day $(\mathrm{SD}=2.65)$ in Groumania, and 0.18 $\mathrm{tf} /$ trap/day $(\mathrm{SD}=0.58)$ in Aboisso Comoe in the dry season. In the rainy season, it was $3.64 \mathrm{tf} / \mathrm{trap} /$ day $(\mathrm{SD}=9.76)$ in Kafolo and $1.42 \mathrm{tf} /$ trap/day $(\mathrm{SD}=2.35$ ) in Groumania. These results show that the diversity of tsetse species along the Comoe River depends on the biotope conservation, as illustrated by the gradual disappearance of species such as G. morsitans submorsitans which was not captured during the study. We also confirm that only the major vector of human African trypanosomiasis, G. palpalis s.I., can adapt to humans.

Keywords: Glossina, biodiversity, trypanosomosis, Comoe River, Côte d'Ivoire
Nekpeni E.B., Dagnogo M., Eouzan J.-P., 1989. Détermination de la limite géographique entre deux sous-espèces de glossines en Côte d'Ivoire : Glossina palpalis palpalis (Robineau - Desvoidy, 1830) et G. p. gambiensis (Vanderplank, 1949). Trop. Med. Parasitol., 40 (1) : 12-15

Omoogun G.A., Dipeolu O.O., Akinboade O.A., 1991. The decline of a Glossina morsitans submorsitans belt in the Egbe area of the derived savanna zone, Kwara State, Nigeria. Med. Vet. Entomol., 5 (1): 43-50

Pielou E.C., 1966. The measurement of diversity in different types of biological collections. J. Theor. Biol., 13: 131-44

Rayaissé J.-B., Courtin F., Akoundjin M., César J., Solano P., 2009. Influence de l'anthropisation sur la végétation locale et l'abondance des tsé-tsé au sud du Burkina Faso. Parasite, 16 (1) : 21-28

Rayaissé J.-B., Kröber T., McMullin A., Solano P., Mihok S., Guerin P.M., 2012. Standardizing visual control devices for tsetse flies: West African species Glossina tachinoides, G. palpalis gambiensis and G. morsitans submorsitans. PLoS Negl. Trop. Dis., 6 (2): e1491, doi:10.1371/journal. pntd.0001491

Reid R.S., Kruska R.L., Deichmann U., Thornton P.K., Leak S.G.A., 2000. Human population growth and the extinction of the tsetse fly. Agric. Ecosyst. Environ., 77 (3): 227-236

Rodhain J., 1926. Le recul de la tsé-tsé : Glossina morsitans devant I'occupation européenne au Katanga. Bull. Soc. Pathol. Exot., 19 : 222-235

Simarro P.P., Jannin J., Cattand P., 2008. Eliminating human African trypanosomiasis: Where do we stand and what comes next? PLoS Med., 5(2): e55, doi:10.1371/journal.pmed.0050055

\section{Resumen}

Djohan V., Kaba D., Rayaissé J.-B., Salou E., Coulibaly B., Dofini F., Kouadio K.A.M., Solano P., Menan H. Diversidad temporo-espacial de las glosinas a lo largo del río Comoé, en Costa de Marfil

Con el fin de estudiar la diversidad temporo-espacial de las glosinas vectores de tripanosomiasis en Costa de Marfil, se llevaron a cabo encuestas a lo largo del río Comoé, en tres sitios localizados en zonas eco climáticas diferentes: al norte, al centro y al sur. Estas se llevaron a cabo durante las estaciones seca y lluviosa. En cada sitio y estación, las glosinas fueron capturadas durante cinco días consecutivos, gracias a trampas bi cónicas dispuestas según transeptos perpendiculares al río Comoé. En Kafolo, en sabana, tres especies y sub especies fueron capturadas independientemente de la estación: Glossina palpalis gambiensis, G. tachinoides y G. medicorum. En Aboisso-Comoé y en Groumania, respectivamente en zona forestal y en transición bosque-sabana, únicamente se capturó G. palpalis palpalis. La densidad aparente por trampa, con todas las especies incluidas, fue más importante en Kafolo que en Groumania y Aboisso-Comoé. Fue de 9,48 glosinas (gl)/trampa/ día [desviación estándar $(\mathrm{ET})=26,30$ ] en Kafolo, de 0,79 gl/ trampa/día $(E T=2,65)$ en Groumania y de $0,18(E T=0,58)$ en Aboisso-Comoé durante la estación seca. Durante la estación Iluviosa, fue de 3,64 gl/trampa/día $(E T=9,76)$ en Kafolo y de 1,42 gl/trampa/día $(E T=2,35)$ en Groumania. Estos resultados muestran que la diversidad de las especies de glosinas a lo largo del río Comoé se basa en la conservación del biotopo, así como lo ilustra la desaparición progresiva de ciertas especies como G. morsitans submorsitans que no fue capturada durante este estudio. Confirmamos igualmente que el único vector mayor de la tripanosomiasis humana africana, G. palpalis s.l. es capaz de adaptarse al hombre.

Palabras clave: Glossina, biodiversidad, tripanosomosis, río Comoe, Côte d'Ivoire 